NASA Technical Memorandum 102566

\title{
Mass Transfer From a Sphere in an Oscillating Flow With Zero Mean Velocity
}

Colin K. Drummond

Lewis Research Center

Cleveland, Ohio

and

Frederic A. Lyman

Syracuse University

Syracuse, New York

\footnotetext{
-- April 1990

(NASA-TM-102560) MASS TRANSFER FROM A

N90-20338

SPATRF IN AN TSCILLATINS. FLOW HITH LERO MEAN

VFLOCITY (NASA) $21 \mathrm{P}$ CSCL 200

Unclas

$63 / 34 \quad 0275330$
} 


$-\ldots-1, \quad+\cdots=$

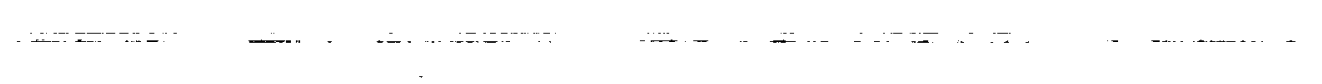

$(-\ldots+\cdots$

$=\ldots .+$

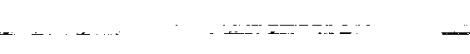




\title{
MASS TRANSFER FROM A SPHERE IN AN OSCILLATING FLOW \\ WITH ZERO MEAN VELOCITY
}

\author{
Colin K. Drummond \\ National Aeronautics and Space Administration \\ Lewis Research Center \\ Cleveland, Ohio 44135 \\ and \\ Frederic A. Lyman \\ Department of Mechanical and Aerospace Engineering \\ Syracuse University \\ Syracuse, New York 13244
}

\begin{abstract}
A pseudospectral numerical method is used for the solution of the Navier-Stokes and mass transport equations for a sphere in a sinusoidally oscillating flow with zero mean velocity. The flow is assumed laminar and axisymmetric about the sphere's polar axis. Oscillating flow results were obtained for Reynolds numbers (based on the free-stream oscillatory flow amplitude) between 1 and 150, and Strouhal numbers between 1 and 1000 . Sherwood numbers were computed and their dependency on the flow frequency and amplitude discussed. An assessment of the validity of the quasi-steady assumption for mass transfer is based on these results.
\end{abstract}

\section{INTRODUCTION}

It is known that under certain conditions the rates of heat and mass transfer are enhanced by the oscillation of the surrounding fluid (Richardson, 1967). This phenomenon is useful in pulsed combustion (Sabnis and Lyman, 1982; Nag and Mukherjee, 1975), drying (Lockwood, 1980), and absorption of high-intensity sound in particle-laden flows (Lyman, 1976; Scott, 1975). Of practical interest is the specific range of Reynolds and Strouhal numbers under which increases in heat and mass transfer can be expected to occur.

Previously, solutions of the flow and mass transfer problems for a sphere in oscillatory flow have been obtained by perturbation techniques for the case in which the fluid displacement amplitude, $A$, is much smaller than the sphere diameter, $d$ (Burdukov and Nakoryakov, 1965; Riley, 1966). In this limit, transport of heat and mass are dominated by acoustic streaming. In the opposite limit, $A / d>>1$, one may use available analytical or experimental results for steady flow and mass transfer, assuming that at each instant a quasi-steady state exists. But in many cases of technological importance, $A / d$ is of order one, and there are no analytical results for that problem. 
The experimental data for mass transfer from spheres were reviewed fourteen years ago by Al-Taweel and Landau (1976), who summarized the numerous Sherwood correlation formulae and attempted a more rational classification of them based (in part) on the $A / d$ ratio. Al-Taweel and Landau suggested that the Sherwood number be correlated with a relation appropriate for acoutic streaming when $A / d<<1$, and that a quasi-steady Sherwood number correlation be used for $\mathrm{A} / \mathrm{d}>0.75$. There is, of course, no reason to expect an abrupt transition at this particular value of $A / d$, and the choice of 0.75 was somewhat arbitrary and based on limited data. Furthermore, the data reviewed were for relatively high Reynolds numbers, because it was convenient to use spheres with diameters on the order of centimeters. For small particles, however, the Reynolds numbers are smaller, and the effect of vibrations on heat and mass transfer at low and moderate Reynolds numbers has received little attention.

The present work was undertaken in order to calculate the rate of mass transfer ${ }^{1}$ from a solid sphere to an oscillating flow in the intermediate range of $A / d(0.1<A / d<2)$ and to investigate the transition from acoustic streaming to quasi-steady transport. This work was intended to complement an experimental program at Syracuse University on combustion of and mass transfer from solid spheres in oscillating flows (Sabnis and Lyman, 1982; Drummond, 1981). The experimental results indicated that an enhancement in burning rate of spherical coal particles was a function of $A / d$ alone up to $A / d=0.94$, whereas the data for mass transfer from sublimating naphthalene spheres, corresponding to $A / d<0.48$, were best correlated by a dimensionless variable appropriate for acoustic streaming

According to dimensional analysis, the Sherwood number can be expressed as a function of $A / d$ (which is the reciprocal of the Strouhal number), the Reynolds number based on the amplitude of the oscillating velocity, and the Schmidt number. Since Schmidt numbers for diffusion into gases (the medium of interest here) are of order one, the most important parameters are the Strouhal and Reynolds numbers. The objective of the present work is to determine the dependence of the Sherwood number on these parameters by numerical solution of the Navier-Stokes and mass transport equations for oscillating flow around a sphere.

\section{FORMULATION OF THE PROBLEM}

The analysis of the unsteady transport problem is simplified by the assumptions that the gas flow is laminar, incompressible, and axisymmetric about the sphere's polar axis ${ }^{2}$, and that the gas and sublimand-laden vapor each behave according to the ideal gas law. The flow field is assumed

1 Computations were also carried out for heat transfer, but to save space the results will not be presented here.

2 It has been observed by Taneda(1956) that even when the flow upstream of a sphere is steady, when the Reynolds number is greater than 130 , the flow is no longer axisymmetrical. 
unaffected by the sublimand motion. The particle temperature is spatially uniform and independent of time, as also are the fluid properties. The effect of natural convection on the transport process is not incorporated in the present problem formulation, but this does not imply the flow is assumed spherically symmetric.

The vorticity transport equation is used to describe the flow field, and only the azimuthal component of vorticity is non-zero for an axisymmetric flow. It is convenient in the numerical analysis to introduce a modified vorticity

$$
\xi=-\exp (z) \zeta \sin \theta
$$

where $\zeta$ is the dimensionless vorticity, related to the dimensional vorticity $\zeta^{*}$, sphere radius $a$ and free-stream velocity amplitude $U_{1}$ by

$$
\zeta=\zeta^{*} a / U_{1}
$$

and $z$ is the contracted radial variable

$$
z=\ln (r / a)
$$

When written in terms of these dimensionless and modified variables, the vorticity transport equation is (see Lin and Lee, 1973):

$$
\frac{M^{2}}{4} \frac{\partial \xi}{\partial \tau}=\frac{R e}{2} \frac{\exp (-3 z)}{\sin \theta}\left(\frac{\partial \psi}{\partial z}\left(\frac{\partial \xi}{\partial \theta}-2 \xi \cot \theta\right)-\frac{\partial \psi}{\partial \theta}\left(\frac{\partial \xi}{\partial z}-2 \xi\right)\right)+\exp (-2 z) D^{2} \xi
$$

where:

$$
\begin{aligned}
& M^{2}=R e \cdot S t \\
& R e=2 U_{1} a / v \\
& S t=2 a \omega / U_{1}
\end{aligned}
$$

and

$$
D^{2}=\frac{\partial^{2}}{\partial z^{2}}-\frac{\partial}{\partial z}+\frac{\partial^{2}}{\partial \theta^{2}}-\cot \theta \frac{\partial}{\partial \theta}
$$

Here, $\psi$ is the streamfunction, defined as

$$
\psi=\psi^{*} / U_{1} a^{2}
$$

and satisfies the equation 


$$
D^{2} \psi=\xi \exp (2 z)
$$

The dimensionless form of the mass transport equation is (see Chuchottaworn et. al., 1984):

$\frac{S t}{2} \frac{\partial Y}{\partial \tau}+\frac{\exp (-3 z)}{\sin \theta}\left(\frac{\partial \psi}{\partial \theta} \frac{\partial Y}{\partial z}-\frac{\partial \psi}{\partial z} \frac{\partial Y}{\partial \theta}\right)=\frac{2 \exp (-2 z)}{R e \cdot S c}\left(\frac{\partial^{2} Y}{\partial z^{2}}+\frac{\partial Y}{\partial z}+\frac{\partial^{2} Y}{\partial \theta^{2}}+\cot \theta \frac{\partial Y}{\partial \theta}\right)$

where $Y$ is the concentration of the sublimand divided by the value at the surface, $S c=\vee / D$ is the Schmidt number, and $D$ is the diffusion coefficient.

Initial and boundary conditions on the solution in the physical domain $\left(0 \leq z \leq z_{\infty}\right)$ shown in Fig.1a are:

$$
\begin{aligned}
& \tau=0: \quad \psi=\xi=0 \\
& Y=0 \quad 0<z \leq z_{\infty} \\
& Y=1 \quad z=0 \\
& \theta=0, \pi: \quad \psi=\xi=\frac{d \psi}{d \theta}=0, \quad 0<z<z_{\infty} \\
& \psi=0 \\
& \xi=\left(\partial^{2} \psi / \partial z^{2}\right)_{z=0} \\
& Y=1 \\
& z=z_{\infty}: \quad \psi=\frac{1}{2} \exp \left(2 z_{\infty}\right) \sin ^{2} \theta \sin \tau \\
& \xi=0 \\
& y=0
\end{aligned}
$$

Note in Eq.(12f) the absence of the term $\partial \psi / \partial z$ since $v=0$ at $z=0$.

\section{NUMERICAL METHOD}

In the present work the vorticity and mass transport equations are integrated using a combination of the pseudo-spectral and finite-difference methods. The implementation of the pseudo-spectral method (PSM) in this work involves expressing the angular dependence of the streamfunction, vorticity, and species concentration in terms of a truncated Fourier cosine series. Finite differences are used to represent the radial and time derivatives. 
The assumed functions for $\psi$ and $\xi$ are expanded in Fourier series on the sphere as follows (Orszag, 1974):

$$
\left\{\begin{array}{l}
\psi(z, \theta, \tau) \\
\xi(z, \theta, \tau)
\end{array}\right\}=\sin ^{2} \theta \sum_{n=0}^{\infty}\left\{\begin{array}{l}
F_{n}(z, \tau) \\
G_{n}(z, \tau)
\end{array}\right\} \cos n \theta
$$

These series automatically satisfy the boundary conditions along the polar axis. When the series in Eq.(13) are truncated at $n=N-1$ and evaluated at the polar angle $\theta_{k}$, one obtains:

$$
\left\{\begin{array}{l}
\psi_{k} \\
\xi_{k}
\end{array}\right\}=\left\{\begin{array}{l}
\psi\left(z, \theta_{k}, \tau\right) \\
\xi\left(z, \theta_{k}, \tau\right)
\end{array}\right\}=\sin ^{2} \theta_{k} \sum_{n=0}^{N-1}\left\{\begin{array}{l}
F_{n}(z, \tau) \\
G_{n}(z, \tau)
\end{array}\right\} \cos n \theta_{k}
$$

The set of coefficients $F_{\mathrm{n}}(z, \tau)$ and $G_{\mathrm{n}}(z, \tau)$ represent $\psi$ and $\xi$, respectively, in spectral space. The transformation between physical space and spectral space is achieved by first selecting a set of evenly spaced collocation points in the polar angle $\theta$ (Figure 1),

$$
\theta_{k}=\frac{\pi}{N}\left(k+\frac{1}{2}\right), \quad 0 \leq k \leq N-1
$$

where $N=\pi / \Delta \theta$. The purpose of the shift by $\pi / 2 N$ is to avoid numerical problems associated with the coefficient $1 / \sin \theta \quad$ in Eqs.(4) and (11). The coefficients in Eq.(14) are determined by the usual inversion of a Fourier series (Gottlieb and Orszag, 1977)

$$
\left\{\begin{array}{l}
F_{n} \\
G_{n}
\end{array}\right\}=\frac{2}{N \delta_{n}} \sum_{k=0}^{N-1}\left\{\begin{array}{l}
\psi_{k} \\
\xi_{k}
\end{array}\right\} \frac{\cos n \theta_{k}}{\sin ^{2} \theta_{k}}
$$

where

$$
\delta_{n}=\left\{\begin{array}{ll}
0, & n<0 \\
2, & n=0 \\
1, & n>0
\end{array}\right\}
$$

This result is used to compute the necessary derivatives and their products in the vorticity transport equation, shown in Eqn.(4). The $z$ - derivatives are approximated by second-order central difference expressions. When a first-order forward difference is used for the time derivative in Eqn.(4), then the vorticity transport equation can be written in the form

$$
\xi^{\tau+\Delta \tau}-\xi^{\tau}=\alpha \Delta \tau \Lambda\left(\xi^{\tau+\Delta \tau}\right)+(1-\alpha) \Delta \tau \Lambda\left(\xi^{\tau}\right)
$$

where $\Lambda(\xi)$ represents the remaining terms in the equation. For $\alpha=0$ the solution is explicit, when $\alpha \neq 0$, implicit. Although test calculations were performed using explicit and implicit 
methods, an explicit method was used for the results presented in the present work. The reason for this was that the value of the weighting factor for convergence changed with time; a factor found to be beneficial at the start of a cycle was potentially detrimental at later times.

The spectral representation of Eq.(10) is given by Orszag (1974):

$$
G_{n} \exp (2 z)=\frac{\partial^{2} F_{n}}{\partial z^{2}}-\frac{\partial F_{n}}{\partial z}-F_{n}(1+n)(2+n)-\frac{6}{\delta_{n}} \sum_{\substack{p=n+2 \\ p+n, \text { even }}}^{N-1} p F_{p}
$$

As before, second-order central difference formulas are used for the $z$ - derivatives. Equation (19) is used to establish the interior values of $F_{\mathrm{n}}$ at the new time step from the (known) boundary values of $F_{\mathrm{n}}$ and the interior $G_{\mathrm{n}}$ at the new time step. An iterative solution was used to solve the resultant matrix equations. No weighting factors were used to accelerate convergence. The solution was assumed to have converged when the difference in the maximum value of $F_{\mathrm{n}}$ was less than 0.005 from one time step to the next (an error on the order of $0.05 \%$ to $0.1 \%$ ); this typically required between 5 and 50 iterations.

Boundary conditions on the sphere surface and in the free stream are enforced with finite differences as shown, for example, in Gottlieb and Orszag (1977). Special attention is drawn to the representation of the vorticity boundary condition at the wall, which in this work is given by

$$
\left.\xi\right|_{z=0}=\frac{\psi_{1, k}-\psi_{0, k}}{2 \Delta z}
$$

Other finite difference approximations for the vorticity boundary condition at the wall were considered (Roache, 1977; Drummond, 1985), but convergence difficulties were experienced with them.

The normalized concentration variable is given by

$$
Y(z, \theta, \tau)=\sum_{n=0}^{N-1} W_{n}(z, \tau) \cos n \theta_{k}
$$

The derivatives of $Y$ required in Eq.(11) are obtained from Eq.(21). Since the computational grid for $Y$ remains unchanged from Fig. 1, the coefficients $W_{\mathrm{n}}$ are computed from

$$
W_{n}=\frac{2}{N \delta_{n}} \sum_{k=0}^{N-1} Y\left(z, \theta_{k}, \tau\right) \cos n \theta_{k}
$$


Substitution of Eq.(21) into Eq.(11) yields the following expression for the mass transport equation. Again, second-order central differences are used for the $z$ - derivatives, and a first-order forward difference used for the time derivative. Once those substitutions have been made, Eq.(11) can be written in the form:

$$
Y^{\tau+\Delta \tau}-Y^{\tau}=\beta(\Delta \tau) L\left(Y^{\tau+\Delta \tau}\right)+(1-\beta) \Delta \tau L\left(Y^{\tau}\right)
$$

The conditions on $\mathrm{Y}$ at the sphere surface and in the free-stream are:

$$
\begin{aligned}
& Y_{0, k}=1,0<k \leq N-1 \\
& Y_{M, k}=0,0<k \leq N-1
\end{aligned}
$$

The boundary conditions along the polar axis are automatically satisfied by the assumed angular dependence of Eq.(21).

We summarize the hydrodynamic and species transport solution procedure selected for use in the present work as follows:

1. Establish the computational mesh with $\theta$ from Eq.(15).

2. Establish initial values of $\psi, \xi, Y$

3. Compute $F_{\mathrm{n}}$ and $G_{\mathrm{n}}$ using Eq.(16).

4. Calculate interior values of $\xi$ at $\tau+\Delta \tau$ from the vorticity transport equation, Eq.(18)

5. Compute new values of $\psi$ at $\tau+\Delta \tau$ from Poissons equation, Eq.(19)

6. Compute new boundary values of $\xi$ from the new interior $\psi_{k}$

7. Compute new $Y$ at $\tau+\Delta \tau$ from the mass transport equation.

8. Repeat steps 3-7 until the solution has converged.

In the explicit calculation procedure of the present work the value of the dimensionless time step was 0.02 . The convergence criterion for mass transfer calculations is discussed below.

\section{RESULTS}

A series of steady flow calculations were made to test the pseudo-spectral code before it was applied to oscillating flow. A drag coefficient of 1.928 for $R e=40$ computed by the PSM on a 31 x 30 grid with $\Delta z=0.07, \Delta \theta=6^{\circ}$, and $z_{\infty}=2.1$, compared favorably with the 1.805 value from the FDM work of Lin and Lee (1973) with $\Delta z=0.1, \Delta \theta=6^{\circ}$, and $z_{\infty}=3.0$. The surface average Sherwood number computed by the PSM was 7.34 for $R e=40$ and $S c=2.54$, which compares reasonably well with the value of 7.65 computed from the correlation of Chuchottaworn et. at. 
(1984). More detailed comparisons are given in Drummond(1985), and a recent discussion of steady flow drag correlations provided in the work of Oliver and Chung (1987). The intent of these computations was simply to compare results of the PSM code to those of the FDM for comparable grids.

\section{Streamfunction}

Oscillating flow computations were made for a vibrational Reynolds numbers (Reynolds numbers based on the free-stream velocity, Eq.6) ranging between 1 and 150, and for Strouhal numbers between 1 and 1000. A typical sequence of the computed instantaneous streamlines is shown in Fig. 2. The lack of published data on the streamfunction or vorticity made an exact comparison of the results impossible, but the streamlines are qualitatively similar to the results of Obakata et. al.(1978) for unsteady flow around a cylinder.

It appears the pseudo-spectral method of analysis of the present work is quite effective since the results of Fig. 2 were obtained with a $21 \times 15$ grid $\left(\Delta z=0.1, \Delta \theta=12^{\circ}, z_{\infty}=2.0\right)$. Several computer runs were made to investigate the time dependence of the results on grid size. Typical results for unsteady flow cases were as follows: the value of the streamfunction at $z=0.2, \theta=$ $90^{\circ}$, and $\tau=1.0$ dropped by $4.6 \%$ when $z_{\infty}$ was extended from 2.0 to 3.5 , and it decreased by $3.1 \%$ when $\Delta \theta$ was reduced from $12^{\circ}$ to $6^{\circ} .^{3}$

Fig.3 illustrates the result obtained by time averaging the streamfunction over one period and is a clear indication of the presence of secondary streaming. Since $A / d$ in Fig. 3 is equal to 1.0 and the Reynolds number equal to 20 , we do not obtain a streaming pattern similar to the results of Riley(1966) whose analysis was for small-amplitude flows $(A / d<<1)$ at low $\left(M^{2}<<\right.$ 1) or high $\left(M^{2}>>1\right)$ frequencies. Although Fig.3 is for $M^{2}=20$, the inner streaming layer is not confined to a region close to the surface. This may be attributed to the fact that $A / d$ is not small. The lines from the center of the sphere to the centers of the closed $\bar{\psi}$ loops in Fig. 3 occur at angles of $45^{\circ}$ to $53^{\circ}$ from the axis of oscillation and radial distances $r / a$ between 2.3 and 2.5 . Lane(1955) calculated that for $A / d<<1$ the center of the inner vortex was at 54043' and $r / a=$ 1.3 , although he cautioned that his streamfunction plots, while accurate in the $\theta$-direction, were very poor approximations in the r-coordinate.

3 As expected, integral quantities are less affected by these grid variations; the surface average Sherwood number, for instance, varied on the order of $0.25 \%$ for the grid extremes described. Given the emphasis on mass transfer for the present study, and the fact that the finer grid was 3.5 times more CPU intensive, the $15 \times 21$ grid was used for most of computations in the present work. 


\section{Mass Fraction Contours}

Mass fraction contours are shown in Fig. 4 at various times over two periods of the freestream velocity, for $R e=100$ and $S t=1$. Similar mass fraction contours were obtained for $R e$ between 10 and 100 and $S t$ between 0.5 and 10 .

\section{Surface-Average Sherwood Number}

The surface average Sherwood number is computed from:

$$
S h=-\int_{0}^{\pi}\left(\frac{\partial Y}{\partial z}\right)_{z=0} \sin \theta d \theta
$$

Fig. 5 illustrates the time dependence of $S h$ for $A / d=1$ and various $R e$ for $\tau$ between 0 and 12 . Because the initial conditions specify $Y=0$ everywhere except at $z=0$, there are initially large gradients of $Y$ at the surface. This is reflected in very high values of $S h$ for $0<\tau<1$. The same is true for the case of $A / d=0.1$, shown in Fig.6. The clear difference in the time dependence of $S h$ for $A / d=1$ and $A / d=0.1$ for $\tau>2.0$ suggests a different mechanism governing mass transport. The oscillatory nature of the Sherwood number in Fig.5 reinforces the idea that at large $A / d$ mass transfer is controlled primarily by the oscillatory flow. Conversely, the profiles of Fig.6 suggest a mass transfer mechanism independent of the primary oscillatory flow, namely that of acoustic streaming. In both cases the effect of $R e$ seems to be primarily to shift the curves to higher or lower $S h$ as opposed to being responsible for any change in the basic mechanism for mass transfer.

The transition in the nature of the time dependence of $S h$ is easier to see when $R e$ is fixed and $A / d$ varied. For the case $R e=20$ and $0.1<A / d<1$, Fig.7 clearly shows the transition from mass transfer controlled by acoustic streaming $(A / d=0.1)$ to mass transfer controlled by the primary oscillatory flow $(A / d=1.0)$. It is not possible to discern from these curves a value of $A / d$ at which the transition occurs. The transition point is best identified from the time-averaged results, as discussed below.

It is necessary to obtain time-asymptotic values of $S h$ in order to compare the results of numerical analysis with predictions of published correlations. Also, since the latter invariably refer to time-averaged mass transfer rates, the time-dependent Sherwood was time-averaged over a period of the free-stream flow oscillation which was sufficiently long after startup so that the starting transient had died out. This procedure was followed regardless of whether the mass transfer process was quasi-steady or due to acoustic streaming. Since the difference in the time averages of the Sherwood number over the ninth and tenth cycles was typically on the order of one percent, the average of the tenth cycle is reported here. 
Fig.8 presents the time-averaged Sherwood number as a function of $A / d$ for various Reynolds numbers from 1 to 150 . The break in each curve corresponds to the transition from acoustic streaming to quasi-steady mass transfer. This occurs at values of $\mathrm{A} / \mathrm{d}$ between 0.1 and 0.25 , considerably lower than the value of 0.75 chosen by A-Taweel and Landau (1976). Also, the $A / d$ at which this transition takes place depends on Reynolds number: at $R e=1$ it happens at $A / d=0.1$, whereas at the higher values $R e=40,100$ and 150 it occurs at $A / d=0.25$, independent of $R e$.

\section{COMPARISON WITH EARLIER WORK AND DISCUSSION}

The time-averaged Sherwood number decreases with increasing $A / d$ at small $A / d$. This behavior is opposite to that predicted theoretically by Burdukov and Nakoryakov (1965) from a boundary-layer analysis in the limit $A / d \rightarrow 0$ such that $M^{2}>>1$. In that limit there is a Stokes shear layer of thickness $\delta=\sqrt{v / \omega}=d / M$ at the surface, as well as a steady acousticstreaming boundary layer of the same thickness. Burdukov and Nakoryakov consider mass transfer due to acoustic streaming only and use the steady velocity at the outer edge of the boundary layer to calculate the convection terms in the convective diffusion equation. Their result for the average Sherwood number over the surface of the sphere can be written

$$
S h=C_{1}\left(U_{1} / \sqrt{\omega \mathcal{D}}\right)=C_{1} \sqrt{R e \cdot S c \cdot A / d}
$$

where $C_{1}$ is a constant of order one ${ }^{4}$. Although the result is written in terms of $A / d$ in the second expression, following Al-Taweel and Landau (1976), this is somewhat misleading, because there is actually no dependence on the sphere diameter. The important parameter in this limiting case is the Reynolds number of the streaming flow

$$
R e_{s t r}=U_{1}^{2} / \omega V=R e \cdot A / d
$$

In a thorough review of oscillatory flows Riley (1967) pointed out that one must carefully distinguish between various possible limiting cases as $A / d \rightarrow 0$. The analysis of Burdukov and Nakoryakov (1965) is not very clear on this point but evidently corresponds to taking the limit in such a way that $R e_{s t r}$ remains finite. According to the above expression for $R e_{s t r}$, this implies that $R e \rightarrow \infty$ as $A / d \rightarrow 0$. The physical relevance of this limit is difficult to imagine, because the assumptions of axisymmetric and laminar flow would be invalid at high $R e$.

The present computations were intended to indicate the transition from acoustic streaming to quasi-steady mass transfer at finite $A / d$ rather than to investigate the limit $A / d \rightarrow 0$. One reason for this is the impossibility of resolving the inner boundary layer when $M>>1$ without using a

4 Burdukov and Nakoryakov (1965) give $C_{1}=1.3$, but there appears to be an error in their calculation of the average over the surface of the sphere. The correct value is $C_{1}=1.89$. 
radial grid spacing for which the computations would become prohibitively expensive. Nevertheless, the computations were carried out down to $A / d=0.001(S t=1000)$, but for fixed $R e$. This corresponds to the physically realizable situation in which the frequency is increased while the velocity amplitude $U_{1}$, sphere diameter and viscosity are held constant. But since $R e_{s t r} \rightarrow \infty$ as $A / d \rightarrow 0$ at fixed $R e$, this limit is quite different from that considered by Burdukov and Nakoryakov, so the computed Sherwood number has a different dependence on $A / d$.

Burdukov and Nakoryakov (1965) also conducted experiments in which camphor balls were exposed to high-intensity, high-frequency acoustic waves. In these experiments $A / d$ was on the order of 0.05 and Reynolds numbers were on the order of 500 . Good agreement between theory and experiment was claimed, but when the correct value $C_{1}=1.89$ is used the agreement is poor.

Padmanabha, Nair, and Ramachandran (1970) measured mass transfer from vertically vibrating naphthalene spheres to air for $0.375<A / d<0.875$ and $200<R e<2000$. Their recommended correlation is

$$
S h=0.63 R e^{0.64}(A / d)^{0.63}
$$

Scatter in the data at low Reynolds number casts doubt on the specific $A / d$ dependence for $R e$ below 300, however. Since the Reynolds number in their experiments and those of Burdukov and Nakoryakov (1965) are well beyond the range $1<R e<150$ of the present computations, it is not possible to make a direct comparison of our results with experiment. In the experiments of Drummond (1981) it was found necessary to provide a steady as well as an oscillatory component of velocity of the air flow to prevent build-up of sublimand in the test section. Since the steady flow introduces additional complications, it was decided to limit the computations to the case of zero mean velocity. 


\section{CONCLUSIONS}

Computations of the flow about and mass transfer from a sphere in oscillatory unsteady flow were carried out with the pseudo-spectal method for values of the Strouhal number between 1 and $1000(A / d$ from 0.001 to 1$)$. The purpose was to obtain accurate solutions of the full Navier-Stokes and convective diffusion equations without the approximations inherent in previous analyses, which were valid only in the limit $A / d<<1$. Since the velocity of the steady secondary flow (acoustic streaming) is typically an order of magnitude less than the primary flow velocity, an indication of the accuracy of the present method is the fact that the time-average of the instantaneous streamfunction gives a flow pattern resembling those calculated or experimentally observed by others. There are still some discrepancies, however, such as the departure of the $\psi$ $=0$ streamline from $\theta=0$ and $90^{\circ}$. In the present work the centers of the inner vortex of the streaming pattern for $S t=1$ occur at an angle of approximately 50 degrees to the axis of oscillation, corresponding well with the theoretical results of Lane(1955) for $A / d<<1$.

To summarize, the effects of the displacement amplitude $A$, velocity amplitude $U_{1}$, and frequency $\omega$ of the oscillatory flow on mass transfer from a sphere of diameter $d$ are as follows:

1. For a fixed $A / d$, mass transfer increases as the Reynolds number $U_{1} d / V$ of the oscillatory flow increases. (This case corresponds to a flow driven by an oscillating piston with fixed stroke but variable frequency).

2. At a fixed Reynolds number, mass transfer decreases as $A / d$ increases (or $\omega$ decreases). Above $A / d=0.25$ mass transfer is virtually independent of $A / d$, at least for the range of Reynolds number considered here $(1<R e<150)$. 


\section{REFERENCES}

1. Al-Taweel,A. and Landau,J.,(1976): Mass transfer between solid spheres and oscillating fluids - a critical review. Canadian J. Chemical Eng. 54, 532-539

2. Baxi,C.B. and Ramachandran,A.,(1969): Effect of vibration on heat transfer from spheres. Journal of Heat Transfer, 91, 337-343.

3. Burdukov,A. and Nakoryakov,V.,(1965): On mass transfer in an acoustic field. J. Applied Mech. and Tech. Physics. 2, 51-55.

4. Chuchottaworn,P., Fujinami,A., and Asano,K.(1984): Numerical analysis of heat and mass transfer from a sphere with surface mass injection or suction. J. Chem. Eng. of Japan. 17, $1-13$.

5. Drummond,C.(1981): Mass transfer from a naphthalene sphere under oscillatory flow. M.S.Thesis, Syracuse University

6. Drummond,C.(1985): Numerical analysis of mass transfer from a sphere in an oscillatory flow. Ph.D. Dissertation, Syracuse University.

7. Gottlieb,D. and Orszag,S.(1977): Numerical analysis of spectral methods. Philadelphia: SIAM Monograph No.26.

8. Lane,C.(1955): Acoustic streaming in the vicinity of a sphere. J. Acoustical Society of America. 27, 1082-1086.

9. Lin,C. and Lee,S.(1973): Transient state analysis of separated flow around a sphere. Computers and Fluids. 1, 235-250.

10. Lockwood,R.(1980): Drying food wastes with pulsating combustion. In: Proceedings for the symposium on Pulse Combustion Technology for Heating Applications. Argonne National Lab. Report ANL/EES TM-87. 224-238.

11. Lyman,F.(1976): Attenuation of high-intensity sound in a droplet laden gas. J. Sound and Vibration. 51, 219-235.

12. Nag,K. and Mukherjee,S.(1975): Effect of pulsation on combustion in fuel beds. Journal of the Institute of Engineering (India) Mech. Eng. Div.. 56, 132-136.

13. Obokata,T., Okajima,A., and Taneda,Y.(1978): Flow and temperature fields around a heated cylinder in a fluctuating flow of Reynolds number - a numerical study on the thermo-fluiddynamic response of the hot-wire. ISAS Report No.566, University of Tokyo. 43, 271-287.

14. Oliver,D. and Chung,J.(1987): Flow about a fluid sphere at low to moderate Reynolds numbers. Journal of Fluid Mechanics. 177, 1-18.

15. Orszag,S.(1974): Fourier series on spheres. Monthly Weather Review. 102, 56-75.

16. Padmanabha,D. Nair,B. and Ramachandran,A.(1970): Effect of vertical vibration on mass transfer from spheres. Indian Journal of Technology. 8, 243-247.

17. Richardson,P.(1967): Effects of sound and vibrations on heat transfer. Applied Mechanics Reviews. 20, 201-217. 
18. Riley,N.(1966): On a sphere oscillating in a viscous fluid. Quart. J. Mech. and Appl. Math.. $16,461-472$.

19. Roache,P.(1977): Computational Fluid Mechanics. Hermosa: Hermosa Publishers.

20. Sabnis,J., and Lyman,F.(1982): Effect of oscillating flow on combustion rate of coal particles. Combustion and Flame. 47, 157-172.

21. Scott,D.(1975): A new approach to the acoustic conditioning of industrial aerosol emissions. J. Sound and Vibration. 43, 605-619.

22. Taneda,S.(1956): Experimental investigation of the wake behind a sphere at low Reynolds number. J. Phys. Soc. Japan. 11, 1104-1114.

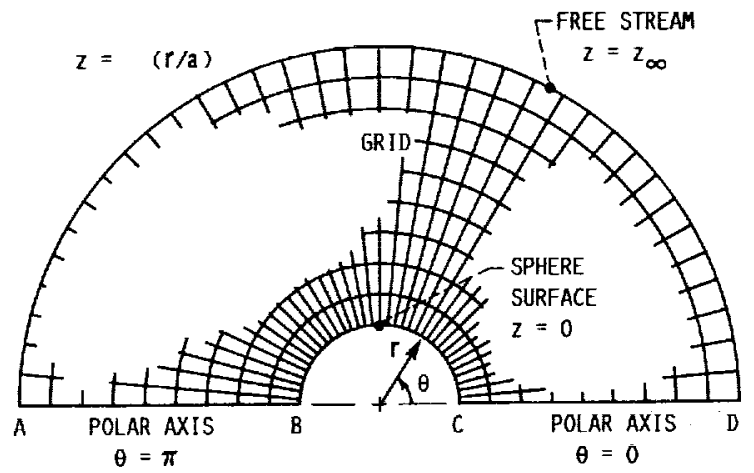

(a) FIELD CONFIGURATION.

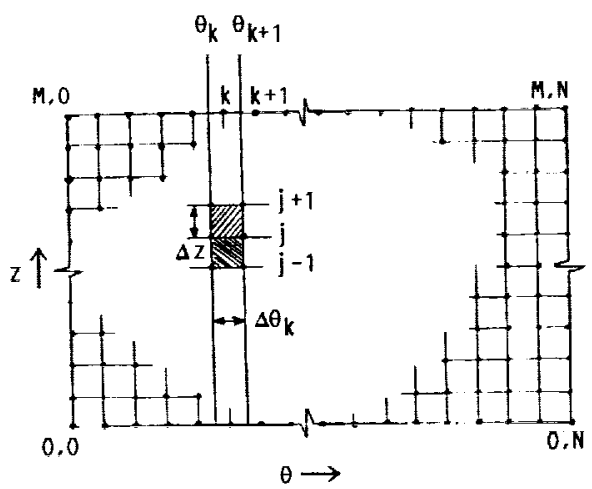

(b) PSM COMPUTATIONAL GRID.

FIGURE 1. - FIELD CONFIGURATION AND PSM COMPUTATIONAL GRID. 


\section{ORIGINAL FAGE IS}
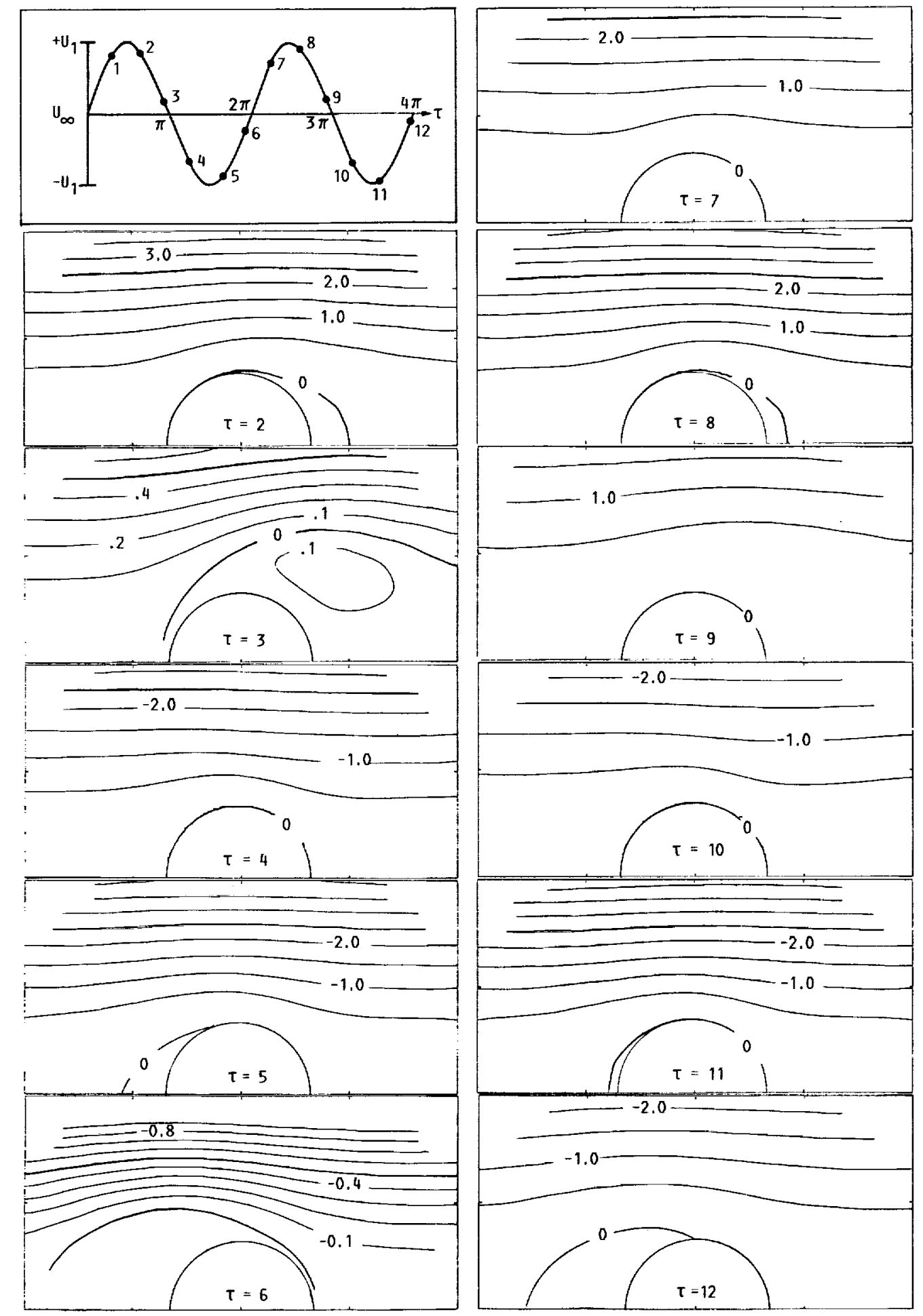

FIGURE 2. - UNSTEADY FLOW STREAML INES FOR AN OSCILLATORY FLOW ABOUT A SPHERE FOR Re $=U_{1} d / v=100$. $S t=\omega d / U_{1}$ FOR VARIOUS VALUES OF THE DIMENSIONLESS TIME $\tau=\omega t$. FREE-STREAM VELOCITY $U_{\infty}=U_{1}$ sin $\tau$. FLOW STARTED FORM REST AT $T=0$. GRID: $\Delta z=0.1, z_{\infty}=2.0 . \Delta \theta=12^{0}$. TIME STEP: $\Delta T=0.02$. 


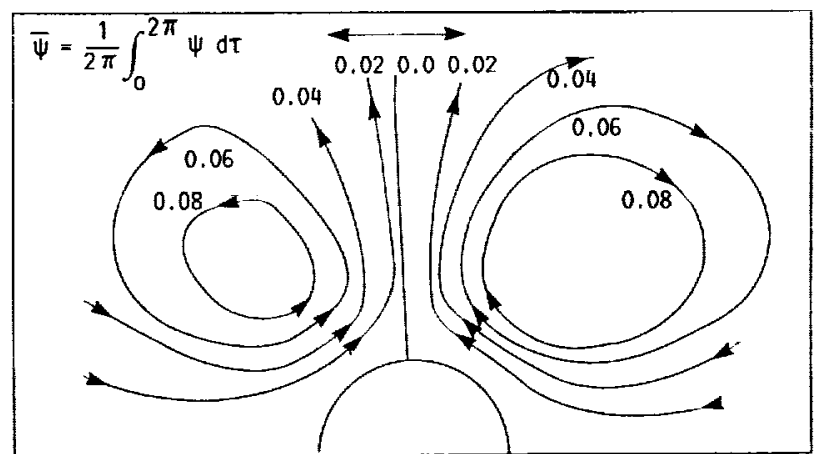

FIGURE 3. - TIME AVERAGED STREAMFUNCTION $\Phi$ FOR Re $=20$ AND $S 1=1$. NOTE THAT A $d=U_{1} / \omega d=1.0($ NOT $\ll 1)$. 


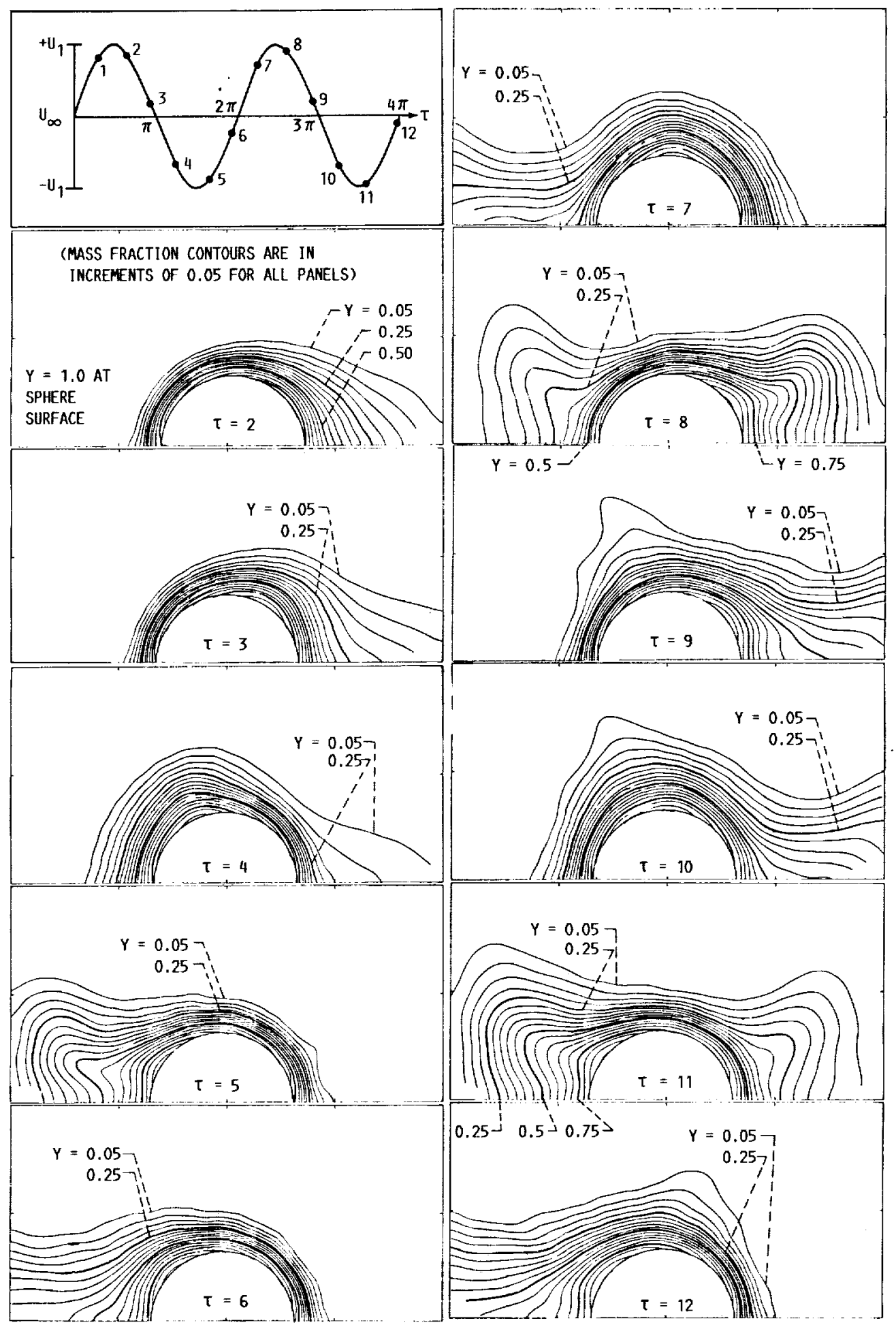

FIGURE 4. - UNSTEADY FLON MASS FRACTION CONCENTRATION CONTOURS FOR OSCILLATORY FLOW AROUND A SPHERE. OTHER CONDITIONS SAME AS FIGURE 2. 


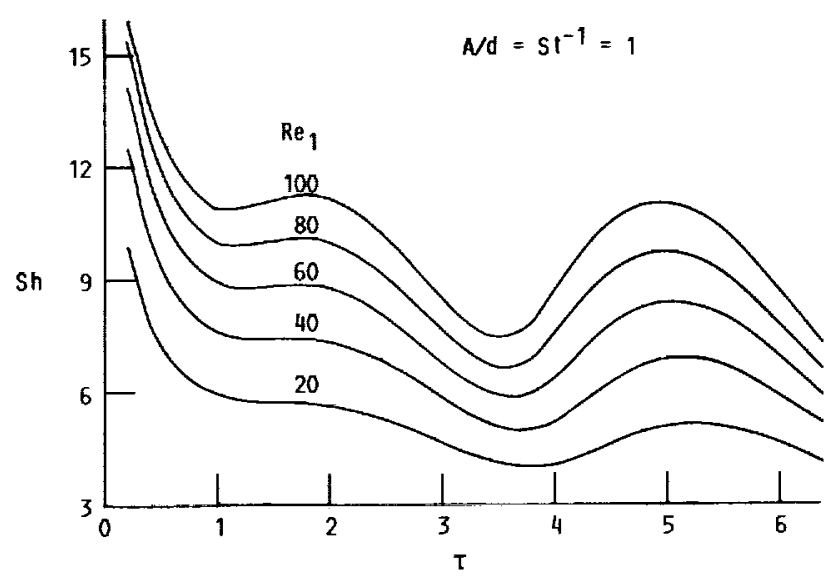

FIGURE 5. - SURFACE AVERAGE SHERHOOD NUMBER AS A FUNCTION OF DIMENSIONLESS TIME $\tau$ FOR A $/ d=1.0$ AND VARIOUS VIBRATIONAL REYNOLDS NUMBERS Re $=U_{1} d / U$.

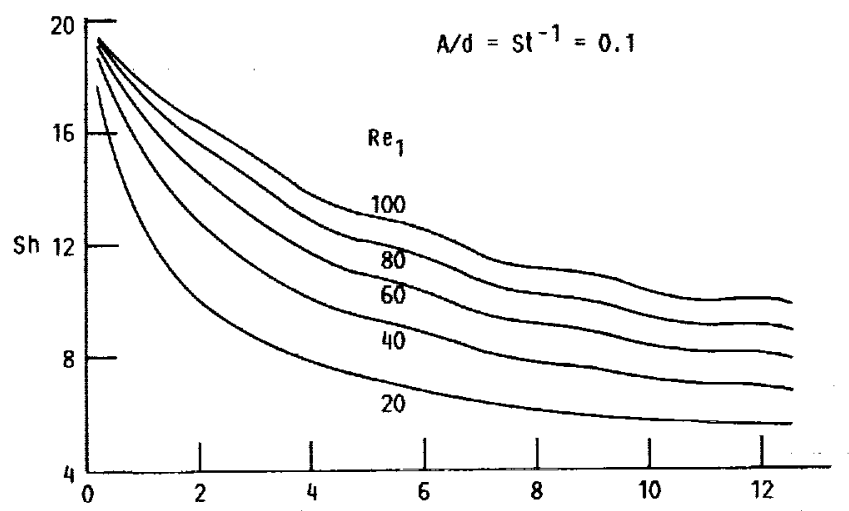

FIGURE 6. - SURFACE AVERAGE SHERHOOD NUMBER AS A FUNCTION OF DIMENSIONLESS TIME $\tau$ FOR A $d=0.1$ AND VARIOUS VIBRATIONAL REYNOLDS NUMBERS Re $=U_{1} \mathrm{~d} / \mathrm{N}$. 


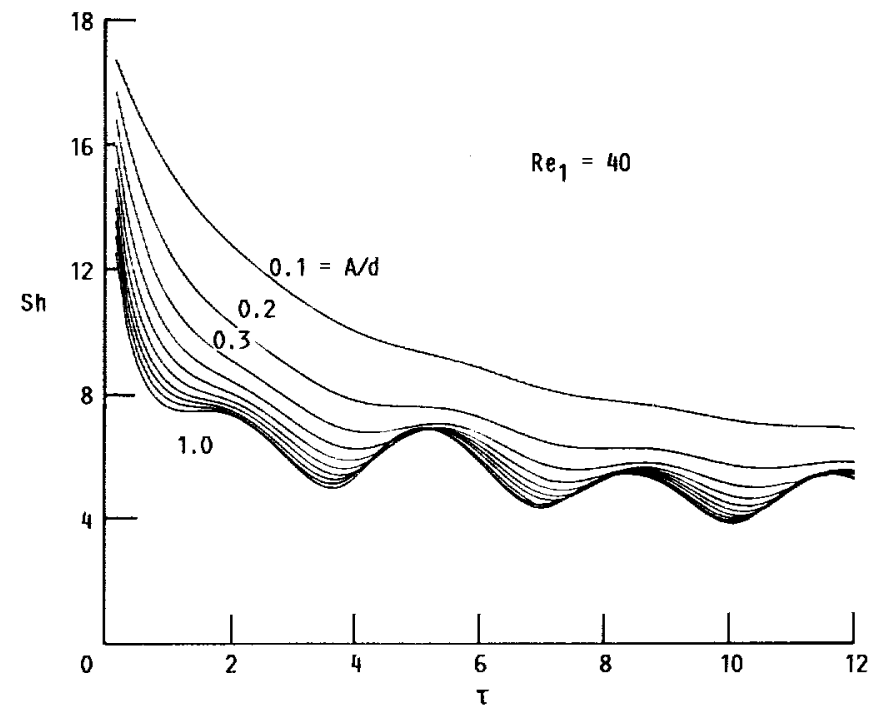

FIGURE 7. - SURFACE AVERAGE SHERHOOD NUMBER AS A FUNCTION OF DIMENSIONLESS TIME $\tau$ FOR A VIBRATIONAL REYNOLDS NUMBERS $R=40$ AND VARIOUS AMPLITUDE $/ D I A M E T E R$ RATIOS $A / d=U_{1} / W d$ $=1 / \mathrm{St}$.

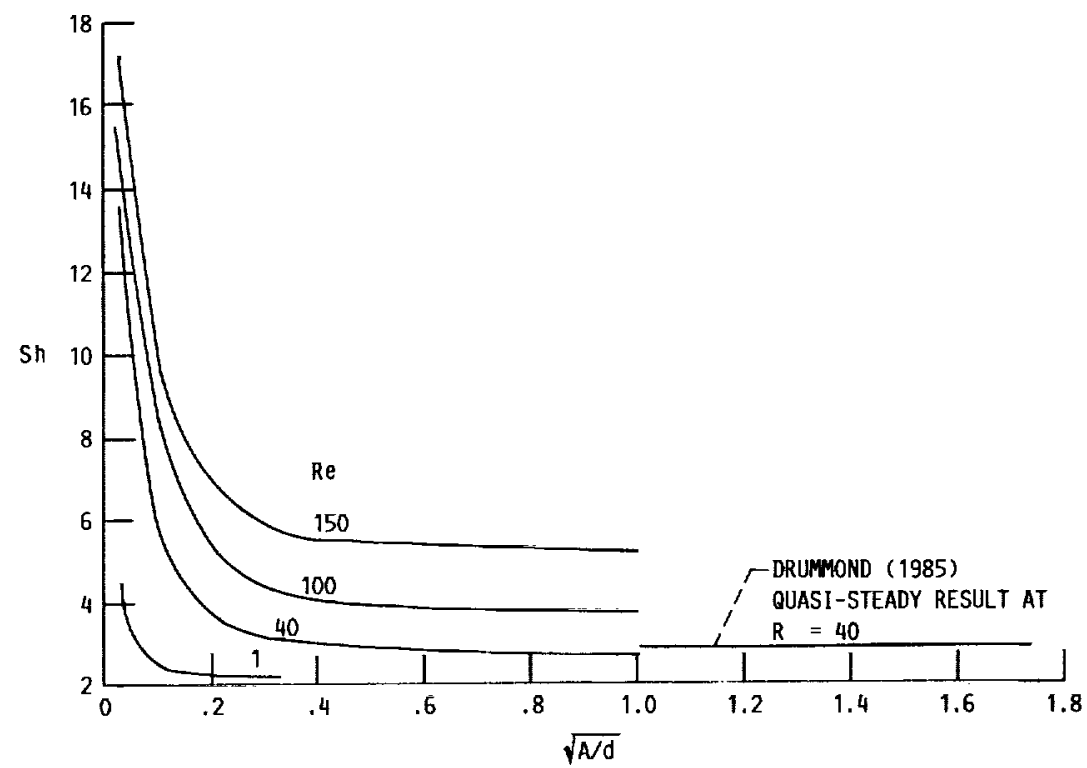

FIGURE 8, - SURFACE AVERAGE, SHERHOOD NUMBER COMPUTED FROM THE PSEUDO-SPECTRAL METHOD FOR VARIOUS VIBRATIONAL. REYWOLDS NUMBERS AND ANd RATIOS. 


\begin{tabular}{|c|c|c|c|c|}
\hline \multicolumn{5}{|c|}{ Report Documentation Page } \\
\hline $\begin{array}{l}\text { 1. Report No. } \\
\text { NASA TM-102566 }\end{array}$ & \multicolumn{2}{|c|}{ 2. Government Accession No. } & \multicolumn{2}{|c|}{ 3. Reciplent's Catalog No. } \\
\hline \multirow{2}{*}{\multicolumn{2}{|c|}{$\begin{array}{l}\text { 4. Title and Subtitle } \\
\text { Mass Transfer From a Sphere in an Oscillating } \\
\text { Flow With Zero Mean Velocity }\end{array}$}} & & \multicolumn{2}{|l|}{$\begin{array}{l}\text { 5. Report Date } \\
\text { April } 1990\end{array}$} \\
\hline & & & \multicolumn{2}{|c|}{ 6. Performing Organization Code } \\
\hline \multirow[t]{2}{*}{$\begin{array}{l}\text { 7. Author(s) } \\
\text { Colin K. Drummond and Fred }\end{array}$} & Lyman & & \multicolumn{2}{|c|}{$\begin{array}{l}\text { 8. Performing Organization Report No. } \\
\text { E-5202 }\end{array}$} \\
\hline & & & \multicolumn{2}{|l|}{$\begin{array}{l}\text { 10. Work Unit No. } \\
505-62-71\end{array}$} \\
\hline \multirow{2}{*}{\multicolumn{3}{|c|}{$\begin{array}{l}\text { 9. Performing Organization Name and Address } \\
\text { National Aeronautics and Space Administration } \\
\text { Lewis Research Center } \\
\text { Cleveland, Ohio } 44135-3191\end{array}$}} & \multicolumn{2}{|c|}{ 11. Contract or Grant No. } \\
\hline & & & \multirow{2}{*}{\multicolumn{2}{|c|}{$\begin{array}{l}\text { 13. Type of Report and Period Covered } \\
\text { Technical Memorandum }\end{array}$}} \\
\hline \multirow{2}{*}{\multicolumn{3}{|c|}{$\begin{array}{l}\text { 12. Sponsoring Agency Name and Address } \\
\text { National Aeronautics and Space Administration } \\
\text { Washington, D.C. 20546-0001 }\end{array}$}} & & \\
\hline & & & \multicolumn{2}{|c|}{ 14. Sponsoring Agency Code } \\
\hline \multicolumn{5}{|c|}{$\begin{array}{l}\text { Colin K. Drummond, NASA Lewis Research Center; Frederic A. Lyman, Department of Mechanical and } \\
\text { Aerospace Engineering, Syracuse University, Syracuse, New York } 13244 .\end{array}$} \\
\hline \multicolumn{5}{|c|}{$\begin{array}{l}\text { 16. Abstract } \\
\text { A pseudospectral numerical method is used for the solution of the Navier-Stokes and mass transport equations for } \\
\text { a sphere in a sinusoidally oscillating flow with zero mean velocity. The flow is assumed laminar and axisymmetric } \\
\text { about the sphere's polar axis. Oscillating flow results were obtained for Reynolds numbers (based on the free- } \\
\text { stream oscillatory flow amplitude) between } 1 \text { and } 150 \text {, and Strouhal numbers between } 1 \text { and } 1000 \text {. Sherwood } \\
\text { numbers were computed and their dependency on the flow frequency and amplitude discussed. An assessment of } \\
\text { the validity of the quasi-steady assumption for mass transfer is based on these results. }\end{array}$} \\
\hline $\begin{array}{l}\text { 17. Key Words (Suggested by Author(s)) } \\
\text { Fluid dynamics } \\
\text { Unsteady flow } \\
\text { Pseudospectral method }\end{array}$ & & $\begin{array}{r}\text { 18. Distribu } \\
\text { Unc } \\
\text { Sub }\end{array}$ & $\begin{array}{l}\text { Unlimited } \\
\text {-Ury } 34\end{array}$ & \\
\hline $\begin{array}{l}\text { 19. Security Classif. (of this report) } \\
\text { Unclassified }\end{array}$ & 20. Securit & $\begin{array}{l}\text { of this page) } \\
\text { assified }\end{array}$ & $\begin{array}{c}\text { 21. No. of pages } \\
20\end{array}$ & $\begin{array}{r}\text { 22. Price* } \\
\mathrm{A} 02\end{array}$ \\
\hline
\end{tabular}

